\title{
Separation and Visualization of Arteries and Heart in 3D Computed Tomography Angiography Images
}

\author{
LAN Shou-ren(兰守忍) ${ }^{1}$, CUI Chao-yi(崔超毅 $)^{2}$, LIU Xin (刘釒) $)^{4}$, JOHNSON Shane ${ }^{3}$, \\ SU Jia-liang(苏佳良) ${ }^{3}$, CHEN Ben-zhi(陈本智) ${ }^{1}$,WANG Li-sheng ${ }^{1 *}$ (王利生)
}

(1. Department of Automation, Shanghai Jiao Tong University, Shanghai 200240, China; 2. Department of Vascular Surgery, Shanghai Ninth People's Hospital, Shanghai Jiao Tong University School of Medicine, Shanghai 200011, China; 3. University of Michigan - Shanghai Jiao Tong University Joint Institute, Shanghai 200240, China; 4. Center for Machine Vision and Signal Analysis, University of Oulu, Oulu 90014, Finland.)

Abstract: The visualization of arteries and heart usually plays a crucial role in the clinical diagnosis, but researchers face the problem of region selection and mutual occlusion in clinical visualization. So the arteries and heart cannot be easily visualized by the current methods of visualization. To solve these problems, we propose a new framework for arteries and cardiac visualization by combining the priori knowledge and set operations. Firstly, a suitable region can be easily determined in transfer function space with the priori knowledge and visual feedback results. Secondly, the arteries and heart can be directly extracted by the marked seed point. Finally, the arteries and heart are separated for solving mutual occlusion through set operations. This framework can easily solve the mutual occlusion problem in clinical visualization and greatly improve the region selection method in transfer function space. Its effectiveness has been demonstrated on the basis of many experimental results when compared with visualization results of clinical medical workstations in real-world 3D computed tomography angiography (CTA) images.

Key words: occlusion removal, multi-atlas, interactive extraction, set operations, volume rendering

CLC number: TP391.9

Document code: A

Received date: 2016-05-19

Foundation item: the National Basic Research program (973) of China (No. 2013CB329401), the National Natural Science Foundation of China (No. 61375020) and the Cross Research Fund of Biomedical Engineering of SJTU (No. YG2013ZD02)

* E-mail: lswang@sjtu.edu.cn 


\section{Introduction}

Volume rendering ${ }^{[1-2]}$ is an important visualization tool, especially useful in medical imaging ${ }^{[3-4]}$ for which the basic requirement is the ability to visualize structures of interest in relation to surrounding structures. An appropriate transfer function must be designed in order to visualize structures of interest in a 3D image ${ }^{[5-13]}$. The appropriate transfer function can assign different voxels with different opacities and colors. So far many transfer functions for vascular and cardiac visualization have also been widely studied by many researchers ${ }^{[14-23]}$. But the arteries, spine and heart are the closely adjacent structures in 3D CTA images, and they are usually occluded by each other in clinical visualization. These structures cannot be easily separated due to the partial volume effects (PVEs) ${ }^{[24]}$; therefore, the previous transfer functions usually fail in visualizing the arteries and heart separately. Although Lan's method ${ }^{[25]}$ can separate and visualize the arteries and heart in volume rendering, it faces the region selection problem in (scalar-gradient transfer function) SG-TF space ${ }^{[8]}$. It is usually done manually by the user through trial-and-error and is a tedious work. The other clinical problem is that the arteries and small vessels cannot be visualized simultaneously.

In this paper, we propose a new framework to greatly improve the region selection method in SG-TF space and solve the mutual occlusion problem in clinical visualization for arteries and cardiac visualization. The proposed framework can provide user-friendly methods to select regions in SG-TF space and extract structures of interest from the determined voxels. Here, we use SG-TF space to determine the voxels of the arteries and heart according to the priori knowledge and visual feedback results. The distribution region of the arteries or heart is the first to be preselected in SG-TF space with the priori knowledge, and then it is slightly adjusted according to the visual feedback results. The second step is to directly extract the arteries or heart from the determined voxels by specifying the seed point in volume rendering. Compared with Lan's method ${ }^{[25]}$, these two steps can greatly improve the region selection method and quickly extract the structures of interest. Finally, the set operations of boundary voxels are still applied to separate the arteries and heart and remove the spine. To some extent, this framework not only improves the region selection method in SG-TF space and quickly extracts structures of interest, but also can separate the aorta and heart because of using set operations. Experimental results further demonstrate the effectiveness of the proposed framework for visualizing the arteries and heart to improve the region selection method in the SG-TF space, while solving the 
mutual occlusion problem in clinical visualization.

\section{Previous Work}

Many researchers have studied the problem of region selection in different transfer function spaces and mutual occlusion in clinical visualization.

For the region selection problem in transfer function spaces, users usually separate different structures of interest by interactively selecting different regions in the transfer function space ${ }^{[5,8,9,11,16 \text {, }}$ ${ }^{18,21,26]}$. But the region selection is usually done manually by the user through trial-and-error, and this is a tedious work. This strategy is not acceptable in clinical visualization.

For the mutual occlusion problem in clinical visualization, the transfer functions for vascular and cardiac system visualization have also been widely studied by many researchers ${ }^{[14-23]}$. Maximum Intensity Projection (MIP) ${ }^{[14-15]}$ was widely used in clinical visualization. But MIP method is gradually abandoned due to lack of depth cues. Surface visualization of the vasculature structure has also been researched ${ }^{[27-28]}$, but researchers face the problem of binary decision and distortions. Compared to surface rendering, volume rendering represents vessels more faithfully, particularly in important changes of the vessel wall. Correa and $\mathrm{Ma}^{[16]}$ proposed a size-based transfer function to explore complex 3D images, but they faced an attributes-overlapping problem in the classification process. Joshi et al. ${ }^{[17]}$ proposed to use the polar profile-based vesselness algorithm to detect vessels, but it is difficult to visualize the aorta and spine separately due to shape similarity. Praßni et al. ${ }^{[18]}$ proposed a shape-based transfer function to avoid performing a voxel level classification and interpreting complex histograms. Läthén et al. ${ }^{[19]}$ presented an approach of automatically tuning transfer functions for visualizing contrast enhanced blood vessels. Selver and Guzelis ${ }^{[20]}$ proposed a semiautomatic method of initial generation of transfer functions to improve the rendering quality for visualizing the tissues of overlapping intensities. These above methods usually fail in visualizing the arteries and spine, two closely adjacent structures, with similar attributes due to PVE; therefore, they also cannot resolve the occlusion problem. Lundstrom et al. ${ }^{[21]}$ proposed local histograms to distinguish iliac artery from pelvic bone in pelvis CTA data. Although this method almost can separate iliac artery and pelvic bone in pelvis CTA data, it cannot separate the aorta and spine of two closely adjacent structures due to PVE. In cardiac system visualization, Zhang et al. ${ }^{[22]}$ proposed real-time visualization of $4 \mathrm{D}$ cardiac MR images, Sera et al. ${ }^{[23]}$ also provided a cardiovascular visualization of high-resolution 4D images, but these two 
methods cannot remove the aorta and spine which are connected to the heart. Although Lan's method ${ }^{[25]}$ can separate and visualize the arteries and heart, the region selection of SG-TF space is very difficult and tedious, and it is time-consuming and tedious to extract the structures of interest from the determined voxels. These shortcomings can bring great inconvenience in clinical visualization.

\section{Method}

In this section, we will discuss how to solve the problem of region selection and mutual occlusion in clinical visualization, as shown in Fig.1.

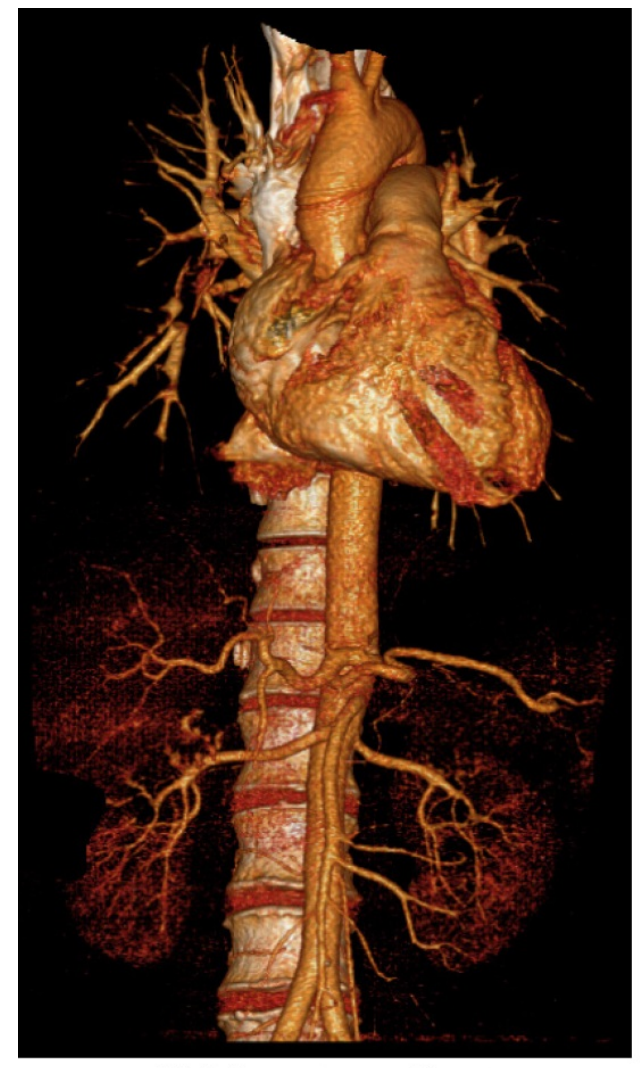

(a) Spine, artery and heart

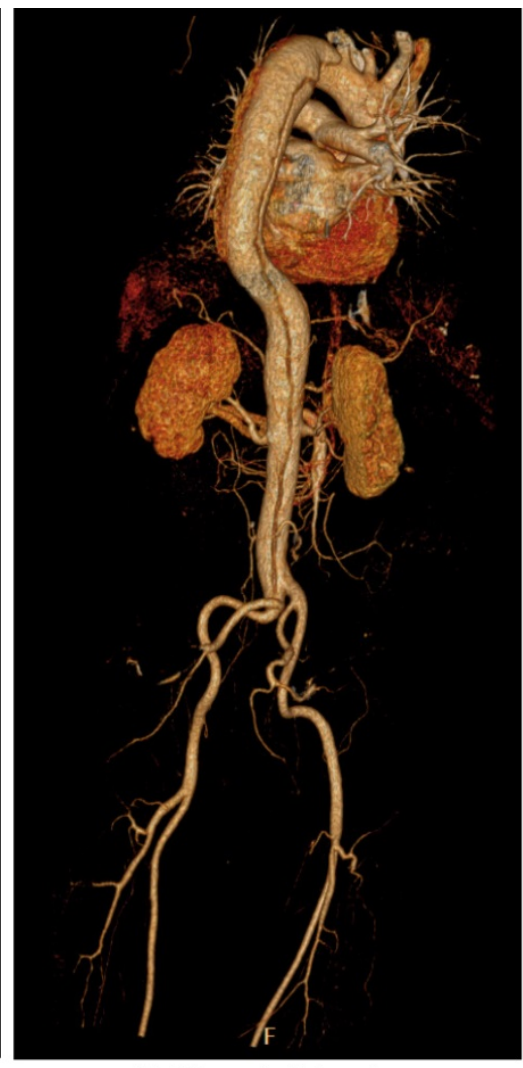

(b) The arterial system

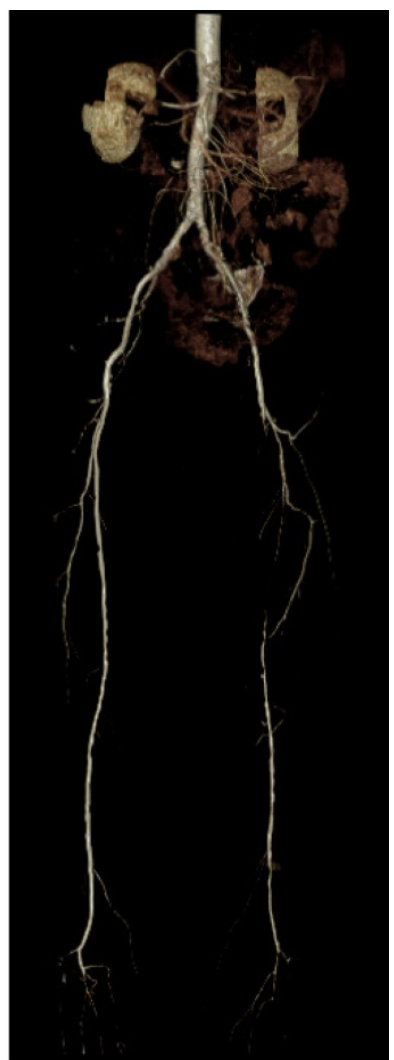

(c) Missing small vessels

Fig. 1 Visualization results of clinical medical workstation from the Shanghai Ninth People's Hospital.

\subsection{Improving the Region Selection Method using the Priori Knowledge in the SG-TF Space}

Generally, the right region of a structure in the SG-TF space is unknown. Currently, the region selection problem of the SG-TF space was also studied in some references ${ }^{[26,29-30]}$. These studies show that the difficult problem of automatically selecting the right region in transfer function space still remains. In this paper, we provide a priori knowledge based method to roughly determine the region, and then we slightly adjust and modify the region in the SG-TF space according to the visual feedback results. This method is very friendly for region selection in SG-TF space when compared with the 
previous method ${ }^{[25]}$. Now, the region selection does not need to be done manually by the user through trial-and-error. Real-time visualization of the structure interest is also implemented in this paper, and we achieve a real-time feedback about visualization results according to the determined region in SG-TF space.

Here, 30 aorta atlases are employed as a priori knowledge to account for the attribute distribution of the scalar and gradient in the SG-TF space. These aorta atlases are from multi-atlas which contains various shapes and attributes of the aorta, and each atlas is a 3D image with manual segmentation of the aorta by the radiologist. The multi-atlas can facilitate us to reduce the error induced by the individual differences of the aorta from patient to patient; therefore it can be used to guide us to classify the aorta from a 3D image. Now it can be freely downloaded from the grand challenge network in biomedical analysis. We find that every atlas has the similar histogram distribution in SG-TF space. The consistency of the distribution of different aorta atlases can provide a unified rule in the choice of region for artery visualization in SG-TF space. In order to remove small differences and specificities, we use an average region model of 30 atlases to roughly determine the region in SG-TF space, as shown in Fig.2(a). The region will be automatically preselected according to the average region model when any CTA image is inputted, as shown in Fig.2(a). Because the aorta and heart have the similar attributes in real-world 3D CTA image, the average region model can also be used as the region model of the heart. Now, we only need to slightly adjust the preselected region $\boldsymbol{U}$ to obtain satisfying voxel sets of the aorta or heart, as shown in Fig.2(b). When we determine the region $\boldsymbol{U}$ from the SG-TF space of a CTA image $f(x, y, z)$ for the aorta or heart, a set of voxels (denoted by $\Phi_{U}$, Eq.(1)) is determined from $f(x, y, z)$. Although $\Phi_{U}$ also contains some unwanted structures and small fragments, they are not all connected spatially, as shown in Fig.2(b).

$$
\Phi_{U}=\{(x, y, z):(f(x, y, z),\|\nabla f(x, y, z)\|) \in U\} .
$$

Where $f(x, y, z)$ is a 3D CTA image, $\boldsymbol{U}$ is the region selected in SG-TF space, $\Phi_{U}$ is a set of voxels determined from $f(x, y, z),\|\nabla f(x, y, z)\|$ represent the gradient magnitude function of $f(x, y, z)$. 


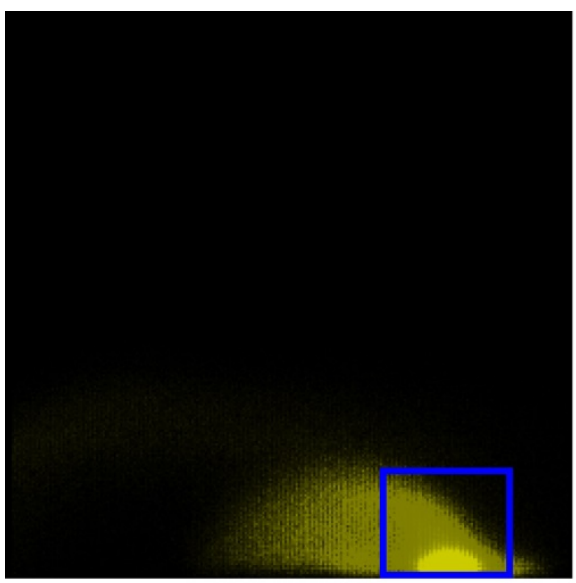

(a) The average distribution of 30 atlases

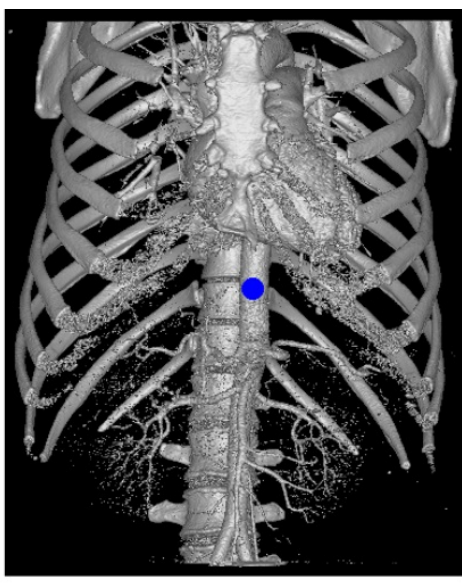

(b) Marked seed point

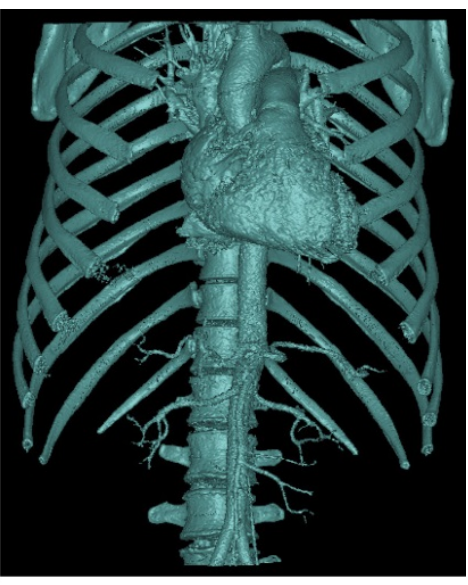

(c) The extracted result

Fig. 2 Interactively extracting the structure of interest using the spatial connectivity in SG-TF space.

\subsection{Extracting the Interesting Structures by Specifying the Seed Point Interactively}

We can easily extract all connected sets from $\Phi_{U}$ by spatial connectivity ${ }^{[25]}$, but it is consuming-time and tedious work in volume rendering. So we modify the spatial connectivity algorithm to directly extract the arteries or heart instead of extracting all connected sets by specifying the seed point on the aorta or heart. To selectively extract the connected set requires a very small amount of time by specifying the seed point. Interactive 3D medical image cutting is widely used as a flexible manual segmentation tool to extract regions of interest in 3D CT images ${ }^{[31]}$. So it has been modified as a seed selection tool for selecting the seed point in volume rendering result of $\Phi_{U}$ in this paper. Taking the aorta as an example, we can interactively mark a seed point on the aorta, as shown in Fig.2(b), and then extract the aorta by the spatial connectivity.

But the aorta, spine and heart are the closely adjacent structures with similar attributes; they are usually mistakenly connected together. Therefore, the aorta, spine and heart are usually extracted simultaneously wherever the seed is selected, as shown in Fig.2(c). This phenomenon usually causes a mutual occlusion problem in clinical visualization, so we need to separate them further for solving the occlusion problem in clinical visualization.

\subsection{Solving the Occlusion Problem using the Set Operations in Clinical Visualization}

The aorta, spine and heart are the closely adjacent structures in 3D CTA images, and they are usually occluded by each other in clinical visualization. Here, we show the separation process for solving the occlusion in a synthetic image. In Fig.3, $S_{1}$ and $S_{2}$ respectively represent the aorta and spine (or heart) in the synthetic image; $\Lambda$ denotes the PVE region between $S_{1}$ and $S_{2}$. We assume that $\Phi=S_{1} \cup S_{2} \cup \Lambda$, as shown in Fig.3(a). Because the scalar values of the voxels in PVE region are 
smaller than those of two adjacent ends of $S_{1}$ and $S_{2}$, we can disconnect $S_{1}$ and $S_{2}$ by removing $\Lambda$ according to the scalar value attribute. Here, the set operations of the boundary voxels ${ }^{[25]}$ are applied to remove $\Lambda$, such as erosion operation, dilation operation and subtraction operation. In this method, the set operations for the separation consist of three main processing steps. Firstly, the erosion operation is applied to remove $\Lambda$ so that $S_{1}$ and $S_{2}$ are disconnected, as shown in Fig.3(b). But $S_{1}$ and $S_{2}$ are eroded as two incomplete boundaries (denoted by $\widetilde{S_{1}}$ and $\widetilde{S_{2}}$, respectively). Secondly, we can dilate $\widetilde{S_{2}}\left(\widetilde{S_{1}}\right)$ in $3 \mathrm{D}$ images so that $\Lambda$ is included in the dilated $\widetilde{S_{2}}\left(\widetilde{S_{1}}\right)$. Denote the dilated $\widetilde{S_{2}}\left(\widetilde{S_{1}}\right)$ by $\overline{S_{2}}$ $\left(\overline{S_{1}}\right)$, as shown in Fig.3(c), where $\overline{S_{1}}=$ the red region $+\widetilde{S_{1}}$ and $\overline{S_{2}}=$ the blue region $+\widetilde{S_{2}}$. Finally, we can subtract the dilated $\overline{S_{2}}\left(\overline{S_{1}}\right)$ from the original $\Phi$ to obtain $S_{1}\left(S_{2}\right)$, as shown in Fig.3(d), where $S_{1}=\Phi-\overline{S_{2}}$ and $S_{2}=\Phi-\overline{S_{1}}$. Note that we first mark two seed regions on $S_{1}$ and $S_{2}$ when performing erosion operation. Our algorithm can know whether two eroded boundaries are separated by judging whether two seed regions belong to two different connected sets in erosion process or not. So the times of erosion operation are adaptive. Additionally, the times of dilation operation are set to 3 here.

Figure 4 intuitively illustrates the process to separate the closely adjacent structures for solving the mutual occlusion. The process includes four steps: (1)determining $\Phi_{U}$ by a priori knowledge and visual feedback adjustment, (2)interactively extracting the aorta by marked seed point according to the spatial connectivity, (3) separating the aorta and spine by the technique in Subsection 2.3, (4) separating the aorta and heart by the technique in Subsection 2.3. These techniques successfully separate the aorta and spine, and separate the aorta and heart. These separated structures are visualized separately, which is conducive to solving the mutual occlusion problem. In conclusion, the correct separation means the solution of the occlusion problem. 


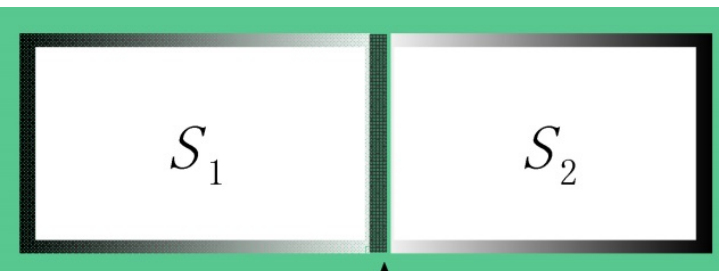

(a)

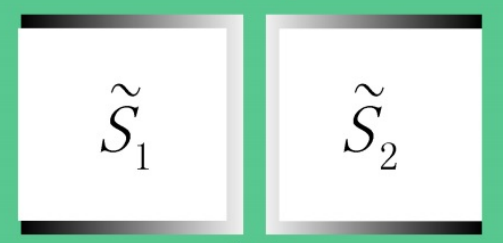

(b)

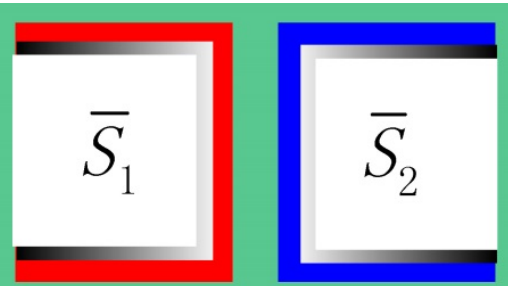

(c)

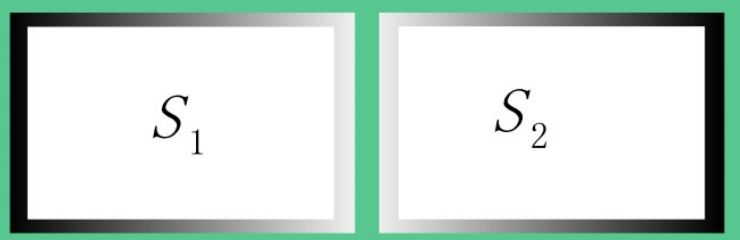

(d)

Fig. 3 Illustrations of the separation process in a synthetic image. (a) Two closely adjacent boundaries and the PVE region. (b) Erosion operation. (c) Dilation operation. (d) Subtraction operation.
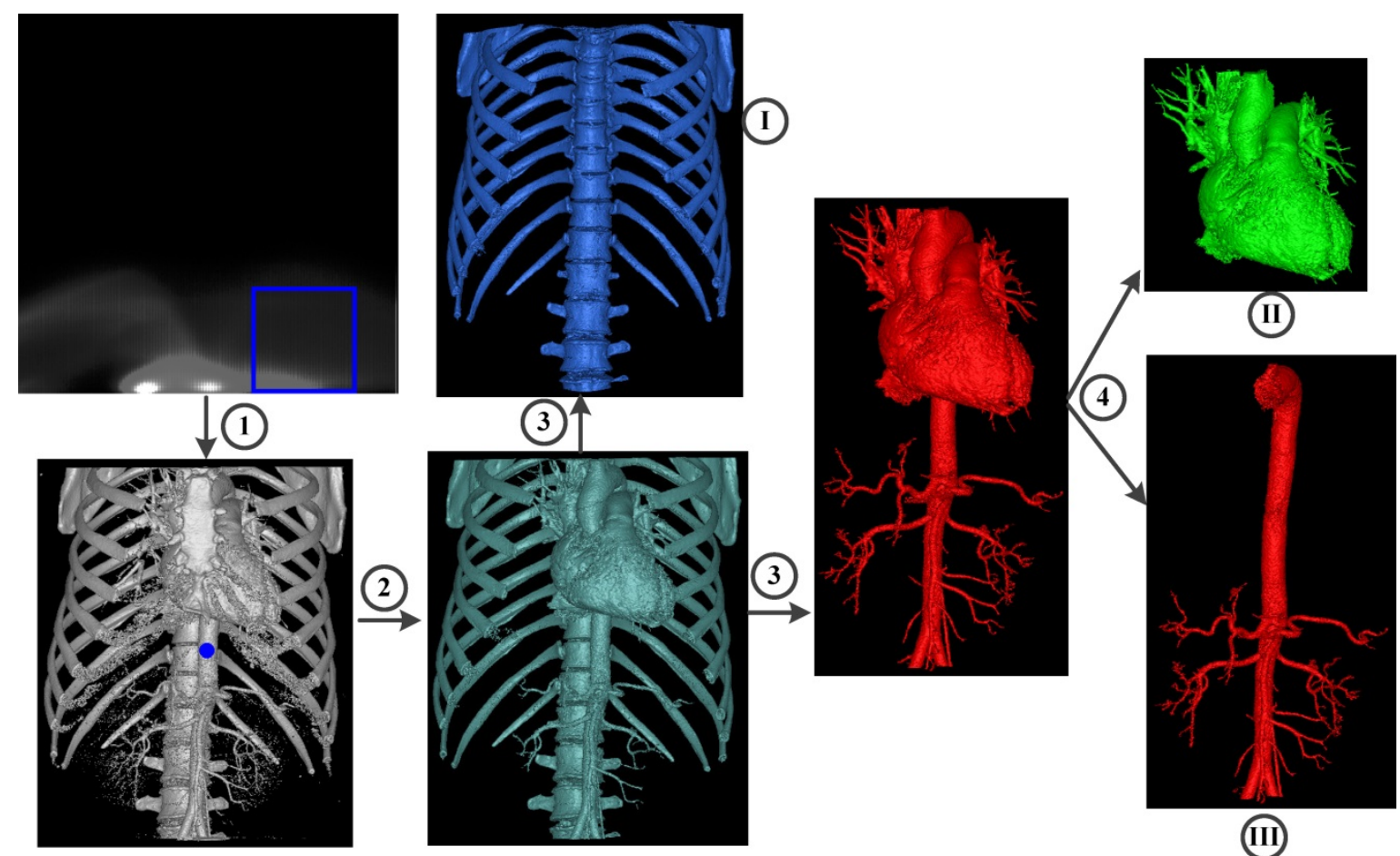

(III)

Fig. 4 An example to illustrate the proposed framework in which three different structures (I - III) are separated from $\Phi_{U}$.

\section{The Clinical Significance and Application}

Occlusion problems usually exist in the departments of vascular surgery and cardiac surgery. There are two kinds of occlusion cases: one is the aorta and spine mutual occlusion, the other is the heart and aorta mutual occlusion. In this section, we discuss the occlusion problem in clinical visualization. 


\subsection{Separation and Visualization of the Aorta and Spine}

In the department of vascular surgery, volume rendering is not often used to display the aorta and the spine separately due to the PVE. So the physician cannot observe the back of the aorta due to the occlusion of spine, as shown in Fig.5(a). However, this phenomenon usually presents a clinically diagnostic inconvenience. To overcome such a problem, we propose a new framework for artery separation and visualization. Now, the aorta and spine can be extracted by the methods in Subsection 2.1-2.2. Then the aorta and spine can be separated by the set operations. The set operations consist of three main processing steps. Firstly, the PVE region is eroded by the erosion operation, and the aorta and the spine are disconnected but they are eroded as two incomplete structures, as shown in Fig.5(b). Secondly, the aorta and the spine are separated by the dilation and subtraction operation in Subsection 2.3, and have a very good visualization quality compared to Fig.5(b), as shown in Figs.5(c) and 5(d). Finally, the physician can rotate the aorta at any angle to observe the aorta in all directions, as shown in Figs.5(d) and 5(e). Although the occlusion problem of the spine is solved, the aorta and heart are still not separated, as shown in Figs.5(d) and 5(e). This occlusion situation is also detrimental to the examination of the aorta and heart. However, the separation of the aorta and heart is still necessary in clinical visualization.

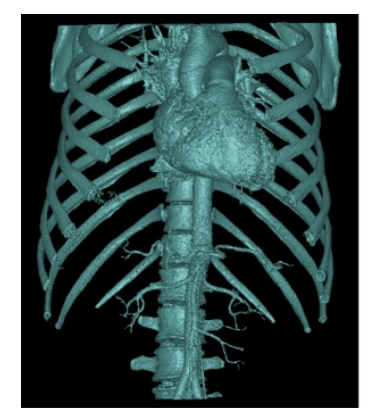

(a) Original boundary

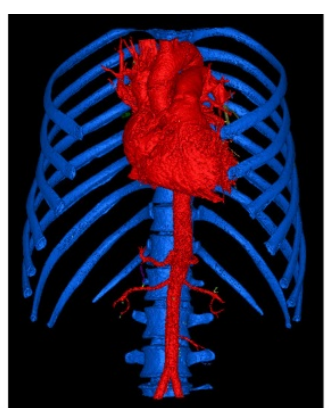

(b) Eroded result

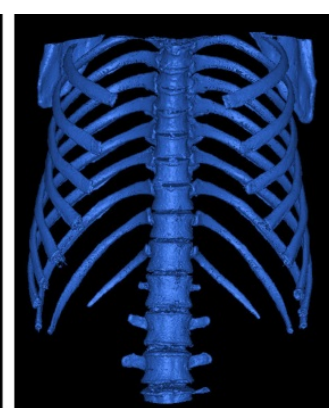

(c) Dilated result

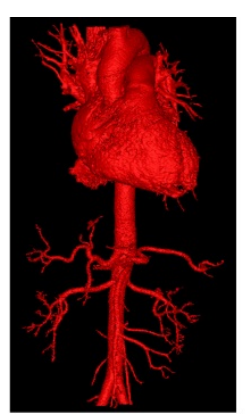

(d) Separated result (e) Another angle

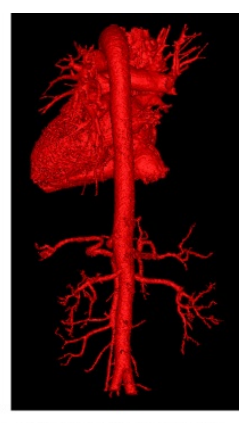

(e) Another angle

Fig. 5 The aorta and spine are separated by set operations in volume rendering and volume rendered in different angles.

\subsection{Separation and Visualization of the Arteries and Heart}

In this section, we introduce the separation of the arteries and heart by the technique in Subsection 2.3. The separation of the arteries and heart is very necessary both in the department of vascular surgery or the department of cardiac surgery, because the arteries are redundant in the department of cardiac surgery, and also the cardiac tissue is superfluous in the department of vascular surgery. Especially in the department of cardiac surgery, the aorta has a serious occlusion impact on the physician's diagnosis. 
Through the statistical validation of different real-world 3D CTA data, we found that there is a gray jump (weak connection) between the aorta and the heart, and the intensity of the junction is smaller than that in other position. Therefore the aorta and the heart can be separated by the technique in Subsection 2.3. Here, the connected arteries and heart are obtained after the aorta and spine are separated according to the method introduced in Subsection 3.1, as shown in Fig.6(a). The separation of the arteries and heart consists of three main processing steps. Firstly, the PVE region is eroded by the erosion operation, and the arteries and the heart are disconnected but they are eroded as two incomplete structures, as shown in Fig.6(b). Secondly, the arteries and the heart are separated by the dilation and subtraction operation in Subsection 2.3, as shown in Fig.6(c). Finally, the physician can rotate the cardiac tissue at any angle to observe the cardiac in all directions, as shown in Fig.6(d).

The most important function is the translucent display; it can preserve the reference information and avoid the occlusion problem in clinical visualization, as shown in Fig.6(d). As for the department of cardiac surgery, the physician can observe the cardiac tissue through the aorta when the aorta is set to be translucent, as shown in Fig.6(d). As for the department of vascular surgery, the physician can observe the arteries through the heart when the heart is set to be translucent, as shown in Fig.6(e). The translucent display has a very good clinical significance; it can keep the reference information while avoiding mutual occlusion, as shown in Figs.6(d) and 6(e).

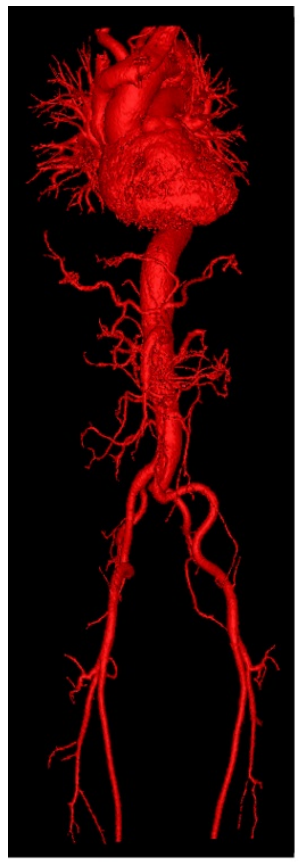

(a)

Original boundary

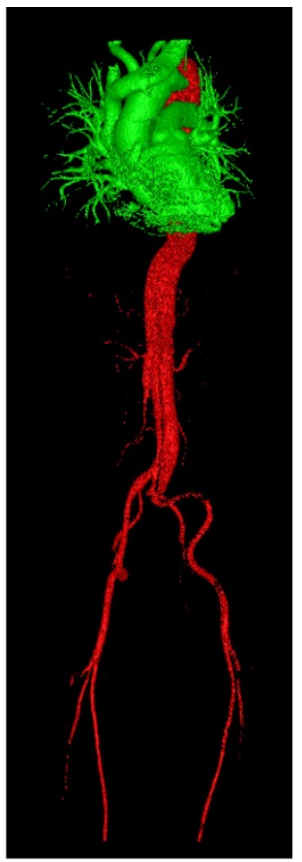

(b)

Eroded result

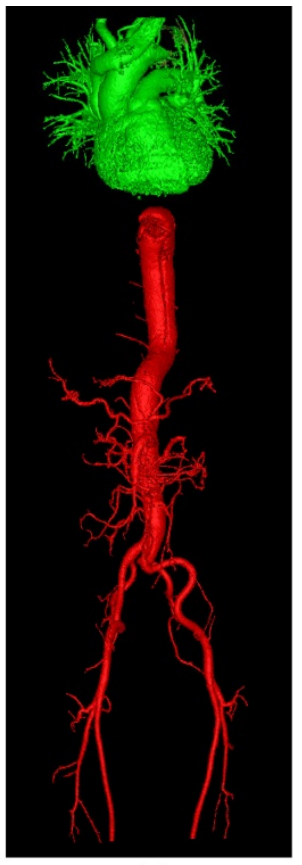

(c)

Separated result

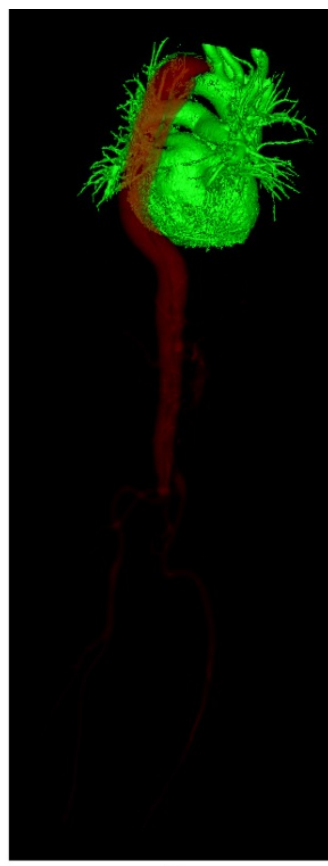

(d)

Semitransparency

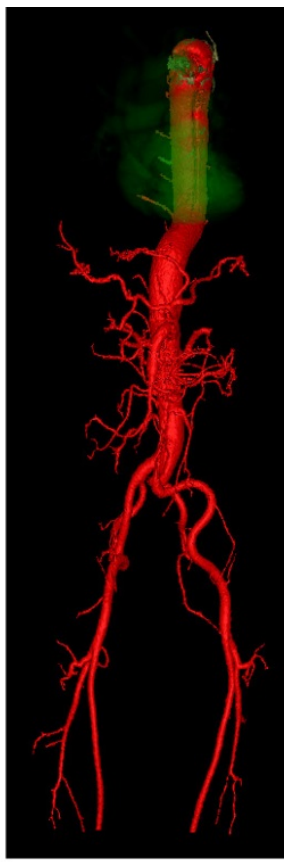

(e)

Fig. 6 The arteries and spine are separated by set operations in volume rendering and volume rendered 
with different opacities and colors in different angles.

\section{Experimental Results and Discussions}

The proposed method has been applied to different 3D CTA images from the Shanghai Ninth People's Hospital. For the comparison with visualization results in Fig.1, experimental results from the same three 3D CTA images are provided in Fig.7. Technique in Section 2 successfully solves the mutual occlusion problem. The spine and arteries can be easily separated, and also the arteries and heart can be easily separated. Different structures in each 3D image are separated and visualized with different colors, as shown in Fig.7(a), Fig.7(b) and Fig.8(d). The visualization results are very beneficial for the physician to observe the arteries and heart in all directions. The physician can set translucence on the arteries and heart or other structures according to different requirements, which means that any structure of interest can be set to be translucent or invisible in Fig.7 and Fig.8.

With the proposed framework in this paper, the clinical occlusion problem is successfully solved, and many small vessels are preserved, as shown in Fig.7 and Fig.8. The details can be compared in Fig.7(c) and Fig.7(d), Fig.8(a) and Fig.8(b), Fig.8(c) and Fig.8(d), Fig.8(e) and Fig.8(f). It is very obvious that our results are better than those of the clinical medical workstation in the terms of the preservation of small vessels. We also take the small vessels as example to give comparisons, as shown in Fig.9. This advantage is very useful in the face of elderly patients and patients with angiostegnosis in department of vascular surgery. Because both elderly patients and patients with angiostegnosis have the narrow vessels like to small vascular structures, if the small vascular structures cannot be detected, it may lead to misdiagnosis.

In conclusion, the proposed framework not only solves the clinical occlusion problem, but also detects small vascular structures. Fig.7 and Fig.8 also demonstrate the ability to solve the clinical occlusion and detect small vascular structures in real-world 3D CTA images. 


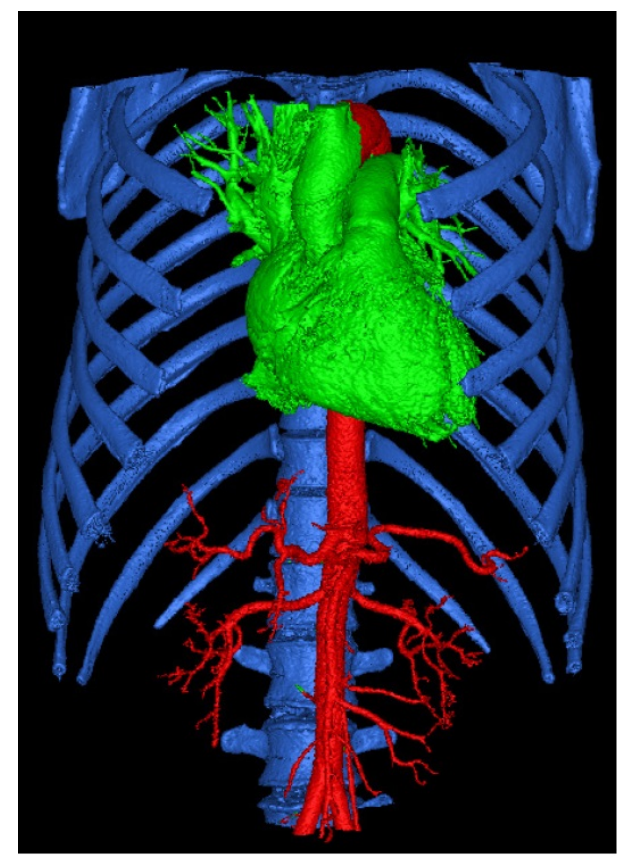

(a) Our result

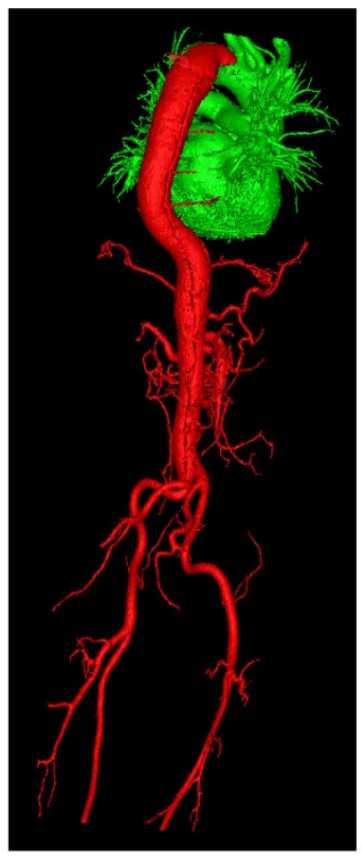

(b) Our result

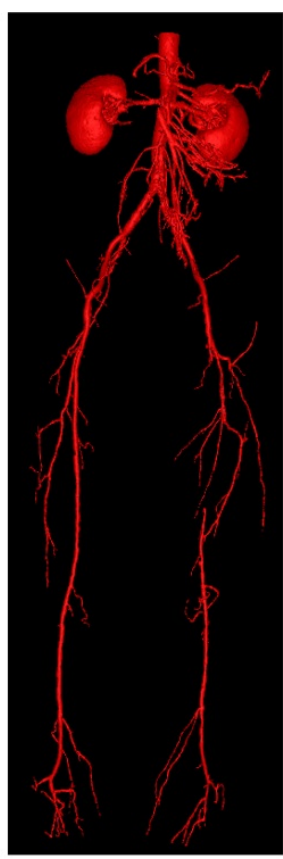

(c) Our result

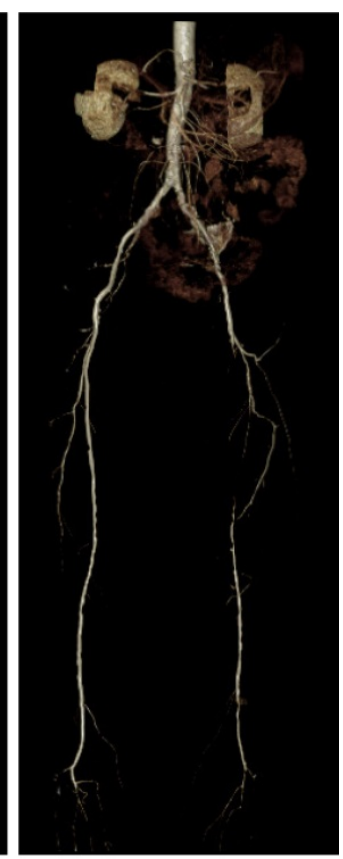

(d) Workstation result

Fig. 7 Visualization of structures contained in the same three 3D CTA data as ones in Fig.1. Different structures are separated by the proposed method and visualized with different colors.

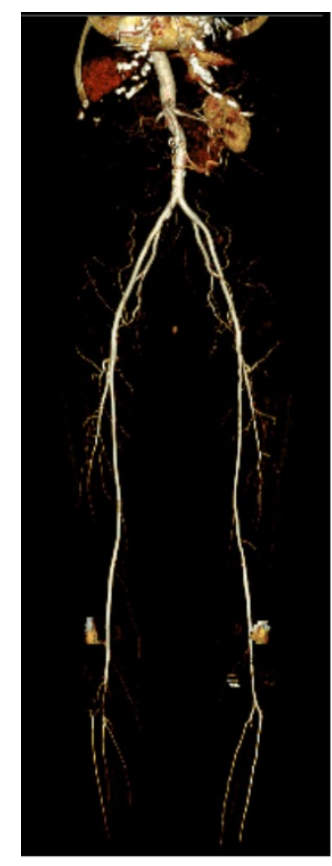

(a) Workstation

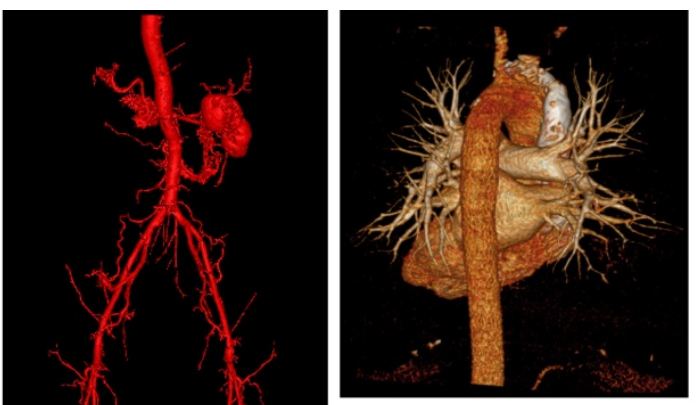

(c) Workstation result

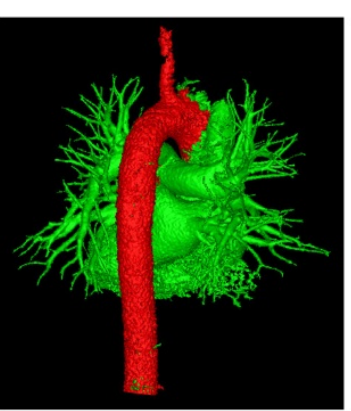

(d) Our result

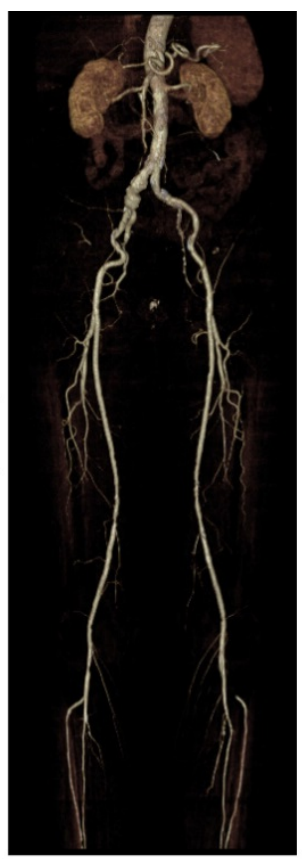

(e) Workstation result

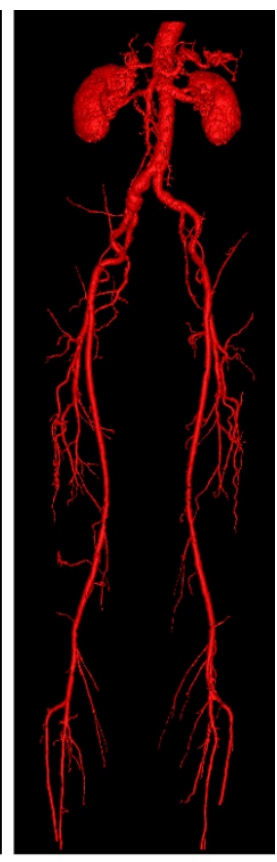

(f) Our result result

Fig. 8 Show more experimental results and comparisons in volume rendering. 


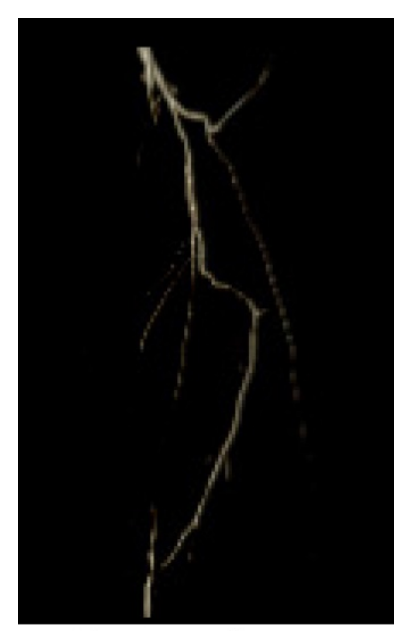

(a) Workstation result

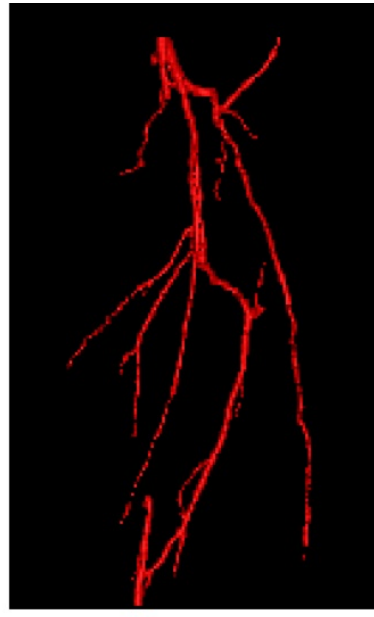

(b) Our result

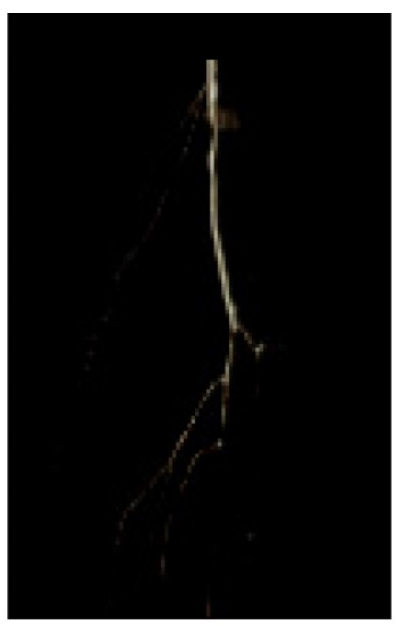

(c) Workstation result

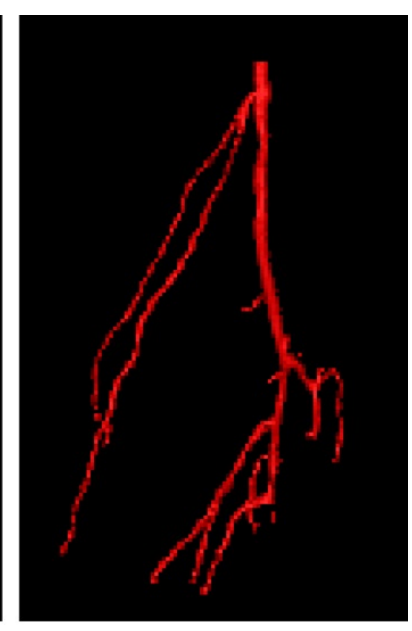

(d) Our result

Fig. 9 Take the small vessels as example to give the results and comparisons.

\subsection{Comparison with the Previous Method}

The differences between the previous method ${ }^{[25]}$ and the proposed framework are obvious and clear. Although Lan's method has a powerful ability to separate different structures with similar attributes in SG-TF space, the region selection of SG-TF space is tedious work, which is usually done manually by the user through trial-and-error. In addition, all connected sets are extracted from the determined voxels $\Phi_{U}$ by previous method, and then the connected set of interest is selected, which is time-consuming. This strategy is not acceptable in clinical visualization. The new framework described in this paper compared with previous work has two novel features, and provide a clinically more acceptable strategy.

The region selection in SG-TF space can be easily determined using the priori knowledge and a preselected region in SG-TF space can be automatically give. Then we can simply adjust the preselected region according to the visual feedback results.

The structure of interest can be directly extracted from $\Phi_{U}$ by interactively specifying a seed point in the volume rendering results. Only the connected set containing the seed point is directly extracted, instead of extracting all connected sets.

In conclusion, the clinical acceptability and usability are better than those of the previous method.

\section{Conclusion}

The vascular and cardiac visualization is becoming particularly important in clinical diagnosis. But current methods face region selection problems in SG-TF space and mutual occlusion problems in 
clinical visualization. To solve these problems, we propose a new user-friendly framework for visualizing the arteries and heart separately. This paper uses the priori knowledge and set operations to solve the region selection problem in SG-TF space and the occlusion problem in clinical visualization. In addition, the structures of interest are directly extracted by interactively specifying the seed point in volume rendering. We have also asked the physician to evaluate the proposed framework and visualization results to get good feedback. Experimental results further demonstrate the effectiveness of the proposed framework for solving the mutual occlusion in real-world 3D CTA images, while improving the region selection method in the SG-TF space.

\section{References}

[1] LEVOY M. Display of surfaces from volume data [J]. IEEE Computer graphics and Applications, 1988, 8(3): 29-37.

[2] DREBin R A, CARPENTER L, HANRAHAN P. Volume rendering[C]// ACM Siggraph Computer Graphics, ACM, 1988, 22(4): 65-74.

[3] Rubin G D, Beaulieu C F, Argiro V, et al. Perspective volume rendering of ct and mr images: applications for endoscopic imaging[J]. Radiology, 1996, 199(2): 321-330.

[4] JOHNSON P T, HEATH D G, KUSZYK B S, et al. CT angiography with volume rendering: advantages and applications in splanchnic vascular imaging[J]. Radiology, 1996, 200(2): 564-568.

[5] Kindlanann G, DuRKIn J W. Semi-automatic generation of transfer functions for direct volume rendering[C]// Proceedings of the 1998 IEEE Symposium on Volume Visualization, ACM, 1998: 79-86.

[6] SATO Y, Westin C F, Bhalerao A, et al. Tissue classification based on 3d local intensity structures for volume rendering[J]. IEEE Transactions on Visualization and Computer Graphics, 2000, 6(2): 160-180.

[7] Pfister H, LOREnSEn B, BAJAJ C, et al. The transfer function bake-off[J]. IEEE Computer Graphics and Applications, 2001, 21(3): 16-22.

[8] Kniss J, KindlmanN G, HANSEN C. Multidimensional transfer functions for interactive volume rendering[J]. IEEE Transactions on Visualization and Computer Graphics, 2002, 8(3): 270-285.

[9] SEREDA P, BARTROLI A V, SERLIE I W O, et al. Visualization of boundaries in volumetric data sets using lh histograms[J]. IEEE Transactions on Visualization and Computer Graphics, 2006, 12(2): 208-218. 
[10] WU Y, QU H. Interactive transfer function design based on editing direct volume rendered images[J]. IEEE Transactions on Visualization and Computer Graphics, 2007, 13(5): 1027-1040.

[11] WANG L, ZHAO X, KAUFMAN A E. Modified dendrogram of attribute space for multidimensional transfer function design[J]. IEEE Transactions on Visualization and Computer Graphics, 2012, 18(1): $121-131$.

[12] CABAn J J, RhEINGANS P. Texture-based transfer functions for direct volume rendering[J]. IEEE Transactions on Visualization and Computer Graphics, 2008, 14(6): 1364-1371.

[13] Kindlmann G, WhitAKeR R, TASDizen T, et al. Curvature-based transfer functions for direct volume rendering: methods and applications[C]// Visualization, 2003: 513-520.

[14] Anderson C M, SAloner D, Tsuruda J S, et al. Artifacts in maximum-intensity-projection display of mr angiograms[J]. AJR. American Journal of Roentgenology, 1990, 154(3): 623-629.

[15] SATO Y, SHIRAGA N, NAKAJIMA S, et al. Local maximum intensity projection (LMIP: a new rendering method for vascular visualization[J]. Journal of Computer Assisted Tomography, 1998, 22(6): 912-917.

[16] CORREA C, MA K L. Size-based transfer functions: a new volume exploration technique[J]. IEEE Transactions on Visualization and Computer Graphics, 2008, 14(6): 1380-1387.

[17] JOSHI A, QIAN X, DIONE D, et al. Effective visualization of complex vascular structures using a non-parametric vessel detection method[J]. IEEE Transactions on Visualization and Computer Graphics, 2008, 14(6): 1603-1610.

[18] Praßni J S, Ropinski T, MEnSmann J, et al. Shape-based transfer functions for volume visualization[C]// 2010 IEEE Pacific Visualization Symposium (PacificVis), IEEE, 2010: 9-16.

[19] Läthén G, LiNDHOLM S, LENZ R, et al. Automatic tuning of spatially varying transfer functions for blood vessel visualization[J]. IEEE Transactions on Visualization and Computer Graphics, 2012, 18(12): 2345-2354.

[20] SELVER M A, GUZELIS C. Semiautomatic transfer function initialization for abdominal visualization using self-generating hierarchical radial basis function networks[J]. IEEE Transactions on Visualization and Computer Graphics, 2009, 15(3): 395-409.

[21] LundSTROM C, LJUNG P, YNNERMAN A. Local histograms for design of transfer functions in direct volume rendering[J]. IEEE Transactions on Visualization and Computer Graphics, 2006, 12(6): 1570-1579. 
[22] Zhang Q, EAgleson R, Peters T M. Real-time visualization of 4d cardiac mr images using graphics processing units[C]// 3rd IEEE International Symposium on Biomedical Imaging. Nano to Macro, IEEE, 2006: 343-346.

[23] SERA T, YoKota H, FUJISAKI K, et al. Development of high-resolution 4d in vivo-ct for visualization of cardiac and respiratory deformations of small animals[J]. Physics in Medicine and Biology, 2008, 53(16): 4285.

[24] LAidlaW D H, Fleischer K W, BARR A H. Partial-volume Bayesian classification of material mixtures in mr volume data using voxel histograms[J]. IEEE Transactions on Medical Imaging, 1998, 17(1): 74-86.

[25] LAN S, WANG L, SONG Y, et al. Improving separability of structures with similar attributes in 2d transfer function design[J]. IEEE Transactions on Visualization and Computer Graphics, 2016, DOI 10.1109/TVCG.2016.2537341.

[26] Roettger S, Bauer M, Stamminger M. Spatialized Transfer functions[C]// EuroVis. 2005: 271-278.

[27] Hahn H K, PREIM B, SelLE D, et al. Visualization and interaction techniques for the exploration of vascular structures[C]// Visualization, 2001. VIS'01. Proceedings. IEEE, 2001: 395-578.

[28] PREIM B, Oeltze S. 3d visualization of vasculature: an overview[M]// Visualization in medicine and life sciences. Springer Berlin Heidelberg, 2008: 39-59.

[29] WANG Y, ZHANG J, LEHMANN D J, et al. Automating transfer function design with valley cell-based clustering of 2d density plots[J]// Computer Graphics Forum, 2012, 31(3pt4): 1295-1304.

[30] IP C Y, VARSHNEY A, JAJA J. Hierarchical exploration of volumes using multilevel segmentation of the intensity-gradient histograms[J]. IEEE Transactions on Visualization and Computer Graphics, 2012, 18(12): 2355-2363.

[31] NING H, YANG R, MA A, et al. Interactive 3d medical data cutting using closed curve with arbitrary shape[J]. Computerized Medical Imaging and Graphics, 2015, 40: 120-127. 